\title{
REVIEW ON ENHANCEMENT OF SURFACE SEGMENTATION
}

\author{
Haziq Ahmad ${ }^{1}$ and Deepika ${ }^{2}$
}

\begin{abstract}
The surface is most important attribute in many image analysis or computer vision applications. An effective and efficient Surface segmentation method is very useful in applications like analysis of aerial images, biomedical images and seismic images as well as automation of industrial applications. In this paper, we present a survey of current surface segmentation methods.
\end{abstract}

Keywords - Surface, Enhancement, Segmentation, Entropy.

\section{INTRODUCTION}

In computer vision, image segmentation [1] is the process of partitioning a digital image into multiple segments (sets of pixels, also known as super-pixels). The goal of segmentation is to simplify and/or change the representation of an image into something that is more meaningful and easier to analyze. Image segmentation is typically used to locate objects and boundaries (lines, curves, etc.) in images. More precisely, image segmentation is the process of assigning a label to every pixel in an image such that pixels with the same label share certain characteristics.

The result of image segmentation is a set of segments that collectively cover the entire image, or a set of contours extracted from the image. Each of the pixels in a region are similar with respect to some characteristic or computed property, such as color, intensity, or texture. Adjacent regions are significantly different with respect to the same characteristic(s). When applied to a stack of images, typical in medical imaging, the resulting contours after image segmentation can be used to create 3D reconstructions with the help of interpolation algorithms like Marching cubes.

Surface [2] means relating to the outer or superficial characteristics, or carrying something on land or sea. An example of surface is the texture of the top of a table. Texture [3] can be termed as a measure of the variation in the intensity of a surface, quantifying properties such as smoothness, coarseness and regularity. It is widely used as a region descriptor in image analysis and computer vision. Surface is characterized by the spatial distribution of gray levels in the neighbourhood of pixels. Resolution at which image is observed determines how Surface is perceived. An effective and efficient Surface segmentation method is very useful in applications like analysis of aerial images, biomedical images and seismic images as well as automation of industrial applications, surface inspection. Surface is qualitatively described as the repetition of the local spatial patterns. Many textural dimensions or parameters are commonly proposed, namely, coarseness, contrast, density, roughness, directionality, frequency, regularity, uniformity, orientation, and so on.

\section{LITERATURE REVIEW}

Yi Zhang et al. [4] discussed that Mental cutting process, a widespread process in the machining, which can produce the maximum number of burrs. Burr detection and deburring are crucially important to safe reliability of parts. In order to avoid the effects of subjective factors effectively, and improve the production efficiency and production automation, we introduced the machine vision technique. According to the universal burrs produced in the cutting process, this paper principally studied the image segmentation, burrs feature extraction, the improvement of adaptability based on digital image processing. The authors conclude that the algorithm applied in this paper can detect the burrs information effectively, laid a solid foundation for automatic polishing, with the certain practical value.

Ichitaro Yamazaki et al.[5] discussed that Extracting features from point-based representations of geometric surface models is becoming increasingly important for purposes such as model classification, matching, and

${ }^{1}$ Department of Electronics and Communication Engineering SVIET, Banur, India

${ }^{2}$ Faculty of Electronics and Communication Engineering SVIET, Banur, India 
exploration. In an earlier paper, we proposed a multiphase segmentation process to identify elongated features in point-sampled surface models without the explicit construction of a mesh or other surface representation. The preliminary results demonstrated the strengths and potential of the segmentation process, but the resulting segmentations were still of low quality, and the segmentation process could be slow. In this paper, we describe several algorithmic improvements to overcome the shortcomings of the segmentation process.

To demonstrate the improved quality of the segmentation and the superior time efficiency of the new segmentation process, we present segmentation results obtained for various point-sampled surface models. We also discuss an application of our segmentation process to extract ridge-separated features in point-sampled surfaces of CAD models.

Segmenting a surface model into its distinct parts is crucial for several applications, such as modeling [6], metamorphosis [7,8], compression [9], simplification [10], retrieval [11,12], collision detection [27], Surface mapping [13], and skeleton extraction [14,15]. Besides our earlier work [4], numerous surface segmentation methods have been developed based on techniques from computer vision [16], load partitioning in finite element methods (FEM) [17], point set clustering in statistics [18], and machine learning [19].

Based on their objectives, segmentation methods can be broadly classified into two categories: patch-type and part-type methods [20]. Patch-type methods obtain segments that are topological disks [21-22], whereas part type methods partition a surface into segments that correspond to features [23, 24-32]. Our methods compute part-type segmentations.

Zhang et al. [30] proposed a feature-based approach for computing a patch-type mesh segmentation for surface parameterization. Their approach identifies a feature by growing a region from a local maximum of the average geodesic distance function and searching for a feature boundary which results in an abrupt increase in size of the surrounded region. Another effective patch type segmentation method, called multi-chart image geometry method (MCIGM), was proposed by Sander et al. [17]. It is based on a k-means algorithm to minimize the global cost of segmentation, where the cost of assigning a face to a segment is measured by the angle between the normal of the face and average normal of all the faces assigned to the segment.

Yamauchi et al. [33] showed that using mean-shift to cluster faces before applying MCIGM makes the method robust against the noise in the input and results in a high-quality segmentation.

Katz and Tal [34] proposed a part-type mesh segmentation method that is based on a k-means algorithm. The cost of assigning a face to a segment is measured by the geodesic distance to the representative face of the segment. The local distance between two connected faces is computed as a weighted sum of their Euclidean and angular distances. Several other successful part-type segmentation methods have been developed based on a watershed technique. This technique locates the negative curvature minima that correspond to segmentation boundaries by simulating the accumulation of water into basins. Even though the segmentation methods discussed so far share some similarity with ours, they all assume that a surface is explicitly represented by meshes. Hence, they cannot be applied directly to the point-sampled surface models, in which the connectivity information is not available. On the other hand, our methods operate directly on the input points. For example, the first phase of our segmentation approach to identify features is similar to the watershed technique, but we explicitly identify the saddle points of a discrete function defined over the input points, and assign each point to a segment based on the gradient flow induced by the discrete function.

Dey et al. [35] proposed a region-growing part-type approach to segment point-sampled surfaces. Their method first identifies the local maxima of a discrete function defined over explicitly-computed 3D meshes. These local maxima represent distinct features, and input points are assigned to a feature based on the flows induced over the meshes. The first phase of our segmentation process is similar to this region-growing approach, but we operate directly on the input points. An advantage to working in the lower dimension (of the surface, in comparison to that of the 3D meshes) is that our segmentation process is efficient. As a result, our method is between two and eleven times as fast as theirs, while generating segmentations that are highly similar to those of Dey et al. in terms of quality. Spectral analysis of an affinity matrix has been used to segment images and meshes [36]. We extend these ideas to collect points that together describe a feature on a surface model.

A-Scan methods were firstly introduced by Hee[37] and were popular until 2005.[38,39] The method introduced in Hee was on the basis of variations in intensity and measured retinal and RNFL thickness. Instead of using simple threes holding, this method was based on one-dimensional edge detection in each A-Scan and was looking for the two most effective edges using peak detection. 
Huang[40] used a similar method in hereditary retinal degenerations in experimental animals and humans.

\section{IV.CONCLUSION}

The area of surface segmentation has undergone tremendous growth in recent years. There has been a great deal of activity both in the refinement of previously known approaches and in the development of completely new techniques. Although a wide variety of methodologies have been applied to this problem, there is a particularly strong concentration in the development of feature-based approaches and on the search for appropriate Surface features. In this paper, we presented a survey of current Surface segmentation.

\section{REFERENCE}

[1]. https://en.wikipedia.org/wiki/Image_segmentation

[2]. www.yourdictionary.com/surface

[3]. Taramati S Taji and Deipali V Gore(2013), "Overview of Texture Image Segmentation Techniques", International Journal of Advanced Research in Computer Science and Software Engineering, Volume 3, Issue 12.Michal Haindl and Stanislav Mikes(2008), "Unsupervised Texture Segmentation", Pattern Recognition Techniques, Technology and Applications, pp. 626,I-Tech, Vienna, Austria.

[4]. Yamazaki, V. Natarajan, Z. Bai, B. Hamann, Segmenting point sets, in: SMI '06: Proceedings of the IEEE International Conference on Shape Modeling and Applications 2006 (SMI'06), IEEE Computer Society, Washington, DC, USA, 2006, pp. 4-13.

[5]. T. Funkhouser, M. Kazhdan, P. Shilane, P. Min, W. Kiefer, A. Tal, S. Rusinkiewicz, D. Dobkin, Modeling by example, in: SIGGRAPH '04: ACM SIGGRAPH 2004 Papers, ACM Press, New York, NY, USA, 2004, pp. 652-663.

[6]. A. Gregory, A. State, M. Lin, D. Manocha, M. Livingston, Interactive surface decomposition for polyhedral morphing, Vis. Comput. 15 (9) (1999) 453-470.

[7]. M. Zockler, D. Stalling, H.-C. Hege, Fast and intuitive generation of geometric shape transitions, Vis. Comput. 16 (5) (2004) 241-253.

[8]. Z. Karni, C. Gotsman, Spectral compression of mesh geometry, in: SIGGRAPH '00: Proceedings of the 27th annual conference on Computer graphics and interactive techniques, ACM Press/Addison-Wesley Publishing Co., New York, NY, USA, 2000, pp. 279-286.

[9]. D. Cohen-Steiner, P. Alliez, M. Desbrun, Variational shape approximation, in: SIGGRAPH '04: ACM SIGGRAPH 2004 Papers, ACM Press, New York, NY, USA, 2004, pp. 905-914.

[10]. M. Attene, B. Falcidieno, M. Spagnuolo, Hierarchical mesh segmentation based on fitting primitives, Vis. Comput. 22 (3) (2006) 181-193.

[11]. E. Zuckerberger, A. Tal, S. Shlafman, Polyhedral surface decomposition with applications, Computer and Graphics 25 (5) (2002) 733-743.

[12]. X. Li, T. Toon, T. Tan, Z. Huang, Decomposing polygon meshes for interactive applications, in: I3D '01: Proceedings of the 2001 symposium on Interactive 3D graphics, ACM Press, New York, NY, USA, 2001, pp. $35-42$.

[13]. B. Levy, S. Petitjean, N. Ray, J. Maillot, Least squares conformal maps for automatic texture atlas generation, ACM Trans. Graph. 21 (3) (2002) 362-371.

[14]. S. Biasotti, S. Marini, M. Mortara, G. Patan'e, An overview on properties and efficacy of topological skeletons in shape modelling, in: SMI '03: Proceedings of the Shape Modeling International 2003, IEEE Computer Society, Washington, DC, USA, 2003, p. 245.

[15]. S. Katz, A. Tal, Hierarchical mesh decomposition using fuzzy clustering and cuts, in: SIGGRAPH '03: ACM SIGGRAPH 2003 Papers, ACM Press, New York, NY, USA, 2003, pp. 954-961.

[16]. J. Shi, J. Malik, Normalized cuts and image segmentation, IEEE Trans. Pattern Anal. Mach. Intell. 22 (8) (2000) 888-905.

[17]. K. Schloegel, G. Karypis, V. Kumar, Graph partitioning for high performance scientific simulations, in: Sourcebook of parallel computing, Morgan Kaufmann Publishers Inc., San Francisco, CA, USA, 2003, pp. 491-541.

[18]. A. K. Jain, M. N. Murty, P. J. Flynn, Data clustering: a review, ACM Comput. Surv. 31 (3) (1999) 264-323.

[19]. V. Vapnik, The Nature of Statistical Learning Theory, Springer-Verlag New York, Inc., New York, NY, USA, 1995. 
[20]. A. Shamir, A formulation of boundary mesh segmentation, in: 3DPVT '04: Proceedings of the 3D Data Processing, Visualization, and Transmission, 2nd International Symposium, IEEE Computer Society, Washington, DC, USA, 2004, pp. 82-89.

[21]. M. Garland, A. Willmott, P. S. Heckbert, Hierarchical face clustering on polygonal surfaces, in: I3D '01: Proceedings of the 2001 symposium on Interactive 3D graphics, ACM Press, New York, NY, USA, 2001, pp. 49-58.

[22]. P. Sander, J. Snyder, S. Gortler, H. Hoppe, Texture mapping progressive meshes, in: SIGGRAPH '01: Proceedings of the 28th annual conference on Computer graphics and interactive techniques, ACM Press, New York, NY, USA, 2001, pp. 409-416.

[23]. K. Zhou, J. Synder, B. Guo, H.-Y. Shum, Iso-charts: Stretch-driven mesh parameterization using spectral analysis, in: SGP '04: Proceedings of the 2004 Eurographics/ ACM SIGGRAPH symposium on Geometry processing, ACM Press, New York, NY, USA, 2004, pp. 45-54. 12 Ichitaro Yamazaki et al.

[24]. S. Katz, G. Leifman, A. Tal, Mesh segmentation using feature points and core extraction, Vis. Comput. 21 (8- 10) (2005) 649-658.

[25]. Y. Lee, S. Lee, A. Shamir, D. Cohen-Or, H. P. Seidel, Intelligent mesh scissoring using 3D snakes, in: PG '04: Proceedings of the Computer Graphics and Applications, 12th Pacific Conference on (PG'04), IEEE Computer Society, Washington, DC, USA, 2004, pp. 279-287.

[26]. R. Liu, H. Zhang, Segmentation of 3D meshes through spectral clustering, in: PG '04: Proceedings of the Computer Graphics and Applications, 12th Pacific Conference (PG'04), IEEE Computer Society, Washington, DC, USA, 2004, pp. 298-305.

[27]. A. P. Mangan, R. T. Whitaker, Partitioning 3D surface meshes using watershed segmentation, IEEE Tran. Vis. Comput. Graph. 5 (4) (1999) 308-321.

[28]. G. Patane, M. Spagnuolo, B. Falcidieno, Para-Graph: Graph-based parameterization of triangle meshes with arbitrary genus, Computer Graphics Forum 23 (4) (2004) 783-797.

[29]. S. Shalfman, A. Tal, S. Katz, Metamorphosis of polyhedral surfaces using decomposition, Proc. Eurographics 21 (3) (2002) 219-228.

[30]. E. Zhang, K. Mischaikow, G. Turk, Feature-based surface parameterization and texture mapping, ACM Trans. Graph. 24 (1) (2005) 1-27.

[31]. Y. Zhou, Z. Huang, Decomposing polygon meshes by means of critical points, in: MMM '04: Proceedings of the 10th International Multimedia Modelling Conference, IEEE Computer Society, Washington, DC, USA, 2004, p. 187.

[32]. P. Sander, Z. Wood, S. Gortler, J. Snyder, H. Hoppe, Multi-chart geometry images, in: SGP '03: Proceedings of the 2003 Eurographics/ACM SIGGRAPH symposium on Geometry processing, Eurographics Association, Airela- Ville, Switzerland, Switzerland, 2003, pp. 146-155.

[33]. H. Yamauchi, S. Lee, Y. Lee, Y. Ohtake, A. Belyaev, H. P. Seidel, Feature sensitive mesh segmentation with mean shift, in: SMI '05: Proceedings of the International Conference on Shape Modeling and Applications 2005 (SMI' 05), IEEE Computer Society, Washington, DC, USA, 2005, pp. 238-245.

[34]. D. L. Page, A. Koschan, M. A. Abidi, Perception-based 3D triangle mesh segmentation using fast marching watersheds, in: Proc. IEEE Conf. Computer Vision and Pattern Recognition, Vol. 2, 2003, pp. $27-32$.

[35]. T. K. Dey, J. Giesen, S. Goswami, Shape segmentation and matching with flow discretization, in: Proc. Workshop on Algorithms and Data Structure, 2003, pp. 25-36.

[36]. C. Gotsman, On graph partitioning, spectral analysis, and digital mesh processing, in: SMI '03: Proceedings of the International Conference on Shape Modeling and Applications 2003 (SMI' 03), IEEE Computer Society, Washington, DC, USA, 2003, p. 165.

[37]. Hee MR, Izatt JA, Swanson EA, Huang D, Schuman JS, Lin CP, et al. Optical coherence tomography of the human retina. Arch Ophthalmol. 1995;113:325-32.

[38]. George A, Dillenseger JA, Weber A, Pechereau A. Optical coherence tomography image processing. Invest Ophthalmol Vis Sci. 2000;41:165-73.

[39]. Koozekanani D, Boyer KL, Roberts C. Retinal thickness measurements in optical coherence tomography using a markov boundary model. IEEE Trans Med Imaging. 2001;20:900-16.

[40]. Huang D, Swanson EA, Lin CP, Schuman JS, Stinson WG, Chang W, et al. Optical coherence tomography. Science. 1991;254:1178-81. 